Revue Gouvernance

Governance Review

GOUVERNANCE

(S)

\title{
Institutions informelles et gouvernance de proximité dans l'orpaillage artisanal. Un cas d'étude au Burkina Faso
}

\section{Oumar Sangaré, Patrick Mundler et Lala Safiatou Ouedraogo}

Volume 13, numéro 2, 2016

URI : https://id.erudit.org/iderudit/1039240ar

DOI : https://doi.org/10.7202/1039240ar

Aller au sommaire du numéro

Éditeur(s)

Centre d'études en gouvernance de l'Université d'Ottawa

ISSN

1912-0362 (numérique)

Découvrir la revue

Citer cet article

Sangaré, O., Mundler, P. \& Ouedraogo, L. S. (2016). Institutions informelles et gouvernance de proximité dans l'orpaillage artisanal. Un cas d'étude au Burkina Faso. Revue Gouvernance / Governance Review, 13(2), 53-73.

https://doi.org/10.7202/1039240ar

\section{Résumé de l'article}

$\mathrm{Au}$ cours des dernières décennies, l'orpaillage artisanal a pris une place grandissante dans plusieurs pays de l'Ouest africain. Ce développement de l'orpaillage artisanal, principalement informel, suscite diverses inquiétudes quant à sa soutenabilité sociale et environnementale, même si divers travaux récents montrent qu'il est un facteur important de résilience pour de nombreux ménages ruraux. À partir de l'étude approfondie d'un site d'orpaillage au Burkina Faso, nous montrons que contrairement aux préjugés habituellement véhiculés qui font des sites d'orpaillage des espaces de non-droit, l'organisation du site repose sur une gouvernance de proximité très structurée, même si elle est principalement régie par des règles informelles. Cette gouvernance de proximité reste néanmoins fragile et comporte diverses insuffisances qui limitent la contribution de l'orpaillage artisanal au développement territorial. Son mode d'exploitation reste principalement orienté vers la rentabilité à court terme, rendant le travail dangereux, précaire et au détriment de la durabilité sociale et environnementale. Ainsi, cette gouvernance de proximité reposant à la fois sur le marché et sur les structures de pouvoir coutumières souffre de ne pas être suffisamment articulée à une politique publique structurante, accompagnant et respectant tout à la fois les mécanismes locaux de régulation. 


\title{
Institutions informelles et gouvernance de proximité dans l'orpaillage artisanal. Un cas d'étude au Burkina Faso
}

\author{
Par Oumar Sangaré, Patrick Mundlerii et Lala Safiatou Ouedraogo ${ }^{i i i}$
}

\section{Résumé}

Au cours des dernières décennies, l'orpaillage artisanal a pris une place grandissante dans plusieurs pays de l'Ouest africain. Ce développement de l'orpaillage artisanal, principalement informel, suscite diverses inquiétudes quant à sa soutenabilité sociale et environnementale, même si divers travaux récents montrent qu'il est un facteur important de résilience pour de nombreux ménages ruraux. À partir de l'étude approfondie d'un site d'orpaillage au Burkina Faso, nous montrons que contrairement aux préjugés habituellement véhiculés qui font des sites d'orpaillage des espaces de non-droit, l'organisation du site repose sur une gouvernance de proximité très structurée, même si elle est principalement régie par des règles informelles. Cette gouvernance de proximité reste néanmoins fragile et comporte diverses insuffisances qui limitent la contribution de l'orpaillage artisanal au développement territorial. Son mode d'exploitation reste principalement orienté vers la rentabilité à court terme, rendant le travail dangereux, précaire et au détriment de la durabilité sociale et environnementale. Ainsi, cette gouvernance de proximité reposant à la fois sur le marché et sur les structures de pouvoir coutumières souffre de ne pas être suffisamment articulée à une politique publique structurante, accompagnant et respectant tout à la fois les mécanismes locaux de régulation.

Mots clés : orpaillage artisanal, gouvernance de proximité, institutions informelles

\section{Abstract}

Over the past decades, artisanal mining has become increasingly important in several West African countries. Various recent studies have shown that it represents a crucial livelihood strategy for many rural households. At the same time, the development of artisanal mining, which is mostly informal, has raised concerns about its social and environmental sustainability.

Based on a detailed study of a small-scale mining site in Burkina Faso, we show that, contrary to what is often assumed, artisanal mines are not places of lawlessness. The mining site's organization depends on proximity-based governance structures even if the rules themselves are informal. However, these governing structures remain fragile and have various shortcomings, which limit the contributions that artisanal mining can make to territorial development. Operations are mostly focused on short-term profitability at the expense of social and environmental sustainability, resulting in dangerous and precarious working conditions. Consequently, these local forms of governance, which are shaped by both markets and traditional power structures, suffer from a lack of meaningful public policies that could accompany, as well as respect, the local regulatory mechanisms in place.

Keywords: artisanal mining, proximity-based governance, informal institutions 


\section{Introduction}

$\mathrm{Au}$ cours des dernières décennies, le secteur minier de plusieurs pays africains a connu d'importants investissements principalement favorisés par les réformes de la Banque mondiale dans les années 1990 et 2000 et par un assouplissement des codes miniers nationaux. Ces conditions ont attiré de grandes compagnies minières (Banchirigah, 2006), malheureusement sans que cela entraîne un développement significatif des pays dans lesquels ces investissements sont réalisés (Sachs et Warner, 2001 ; Gylfason et Zoega, 2006). Le secteur minier se caractérise également par une croissance de l'orpaillage artisanal qui, selon la Banque mondiale (World Bank, 2009), concerne des centaines de milliers de personnes dans les pays disposant de ressources aurifères.

Au Burkina Faso, l'or est passé en tête des produits d'exportation du pays, devançant le coton et faisant de ce pays le quatrième producteur d'or du continent après l' Afrique du Sud, le Ghana et le Mali (Airault, 2012; Arnaldi di Balme et Lanzano, 2014).

Comme dans d'autres pays, parallèlement aux investissements réalisés par des entreprises multinationales, on constate un intérêt grandissant pour l'orpaillage artisanal au sein des populations locales. Ce développement de l'orpaillage artisanal, principalement informel, est souvent critiqué et suscite diverses inquiétudes quant à sa durabilité. Sur le plan environnemental, l'usage non contrôlé de plusieurs substances chimiques comme le mercure et le cyanure entraîne la pollution des eaux de surface et souterraines (Ouédraogo, 2006). Sur le plan social, divers constats font état d'un accroissement des empoisonnements liés à ces mêmes substances (Tomicic et coll., 2011 ; OMS, 2013). La pratique de l'orpaillage est par ailleurs souvent associée à divers problèmes sociaux : absentéisme scolaire, développement de la prostitution, développement de divers trafics, accroissement de la consommation de drogues et expansion du banditisme (Sawadogo, 2011 ; Yaro et coll., 2011).

Sur le plan socioéconomique, l'orpaillage artisanal se pratique en complément ou en substitution de l'agriculture, ce qui peut mettre en péril l'amélioration de la productivité de l'agriculture, qui reste pourtant le principal secteur d'activités pour $80 \%$ des ménages et fournit des produits déterminants pour la sécurité alimentaire des ruraux (Okoh et Hilson, 2011 ; Hilson et Garforth, 2012). Cela dit, divers travaux montrent aussi que l'orpaillage artisanal, grâce aux revenus qu'il procure, est un facteur majeur de résilience pour de nombreux ménages ruraux (Hentschel et coll., 2002 ; Doucouré, 2014 ; Teschner, 2014). Au Burkina Faso, on estime que l'orpaillage artisanal représente plus de 80 milliards FCFA de revenus pour environ 1,3 million de personnes situées principalement en milieu rural (Maradan et coll., 2011).

À partir de l'étude d'un site d'orpaillage artisanal situé dans la commune rurale de Gbomblora dans la région du sud-ouest du Burkina Faso (Sangaré, 2016), nous allons 
analyser la façon dont diverses institutions, pour l'essentiel informelles, permettent le fonctionnement concret et quotidien du site.

Notre cadre théorique est celui de l'économie de la proximité, concept à la fois relationnel et spatial, qui s'intéresse au caractère territorial des processus productifs et aux effets des proximités géographique et relationnelle sur le développement territorial et sur la façon dont les individus (ou les collectifs) se coordonnent.

L'école de la proximité est née au début des années 1990 (Bellet et coll., 1993) de la réflexion d'un groupe de chercheurs formé d'économistes régionaux intéressés par la mobilisation des outils de l'économie industrielle pour analyser le développement territorial et d'économistes industriels intéressés par le caractère territorial des processus productifs (Torre et Gilly, 1999; Pecqueur et Zimmermann, 2004 ; BoubaOlga et coll., 2008). Ce groupe appelé «dynamiques des Proximités » s'est très vite ouvert à des chercheurs d'autres disciplines (géographie, sociologie) et rassemble, à chaque édition de son colloque bisannuel, plus d'une centaine de chercheurs.

Tous les auteurs de ce courant s'accordent globalement sur ce que recouvre le caractère spatial de la proximité. La proximité géographique traduit la distance kilométrique entre deux acteurs, pondérée par la perception qu'ont les individus de cette distance en termes de temps passé ou de coût de transport (Rallet et Torre, 2004). Lorsque l'on s'intéresse à son rôle dans la coordination des individus ou des organisations, la proximité géographique peut revêtir différentes dimensions. Elle peut être permanente ou temporaire (Torre, 2010), ce qui est le cas des sites d'orpaillage qui sont des lieux occupés de façon permanente, notamment par les villageois, mais aussi de façon saisonnière par une partie des orpailleurs. Elle peut aussi être recherchée ou subie (Torre, 2008; Torre et Beuret, 2012): recherchée lorsqu'elle est activée par les acteurs pour favoriser leur coordination; subie lorsque des acteurs ont à supporter les effets d'une activité (bruits, odeurs, concurrence inéquitable...) sans pouvoir choisir de s'en éloigner.

La nature du caractère relationnel de la proximité suscite en revanche davantage de débats, mais tous les auteurs mettent en avant l'importance des institutions, qu'il faut ici comprendre comme un ensemble de règles et de représentations communes (Gilly et Lung, 2005). Ces institutions sont souvent en mesure d'accompagner les collectifs informels (Filippi et Torre, 2003) ainsi que la structuration de systèmes de gouvernance territoriale (Gilly et Pecqueur, 2000). De ce fait, l'école de la proximité met en avant l'idée que les marchés ne sont pas les seuls outils de coordination, mais que des institutions diverses peuvent servir de cadre à l'organisation d'un monde de production, de distribution et d'échange (Talbot et Kirat, 2005 ; Bouba-Olga et coll., 2008 ; Carrincazeaux et coll., 2008 ; Talbot, 2008 ; Beaurain et coll., 2009). 
Pour traiter des questions de coordination autour du thème de l'orpaillage artisanal, nous reprenons la caractérisation de la proximité relationnelle proposée par Gilly et Torre (2000) ou Zimmerman (2008), qui la nomment proximité organisée. La proximité organisée comprend elle-même deux logiques: la logique d'appartenance et la logique de similitude. La logique d'appartenance se réfère à l'appartenance à une même organisation, vue ici comme «tout ensemble structuré de relations sans préjuger de la forme de la structure (...)» (Rallet et Torre, 2004 : 27). Grâce à cette logique d'appartenance, les individus nouent plus facilement des interactions entre eux. Comme on le verra dans la suite de cet article, cette logique d'appartenance est activée dans le développement d'un site d'orpaillage par la rencontre entre un promoteur, un propriétaire terrien, les autorités coutumières villageoises et des orpailleurs. La logique de similitude ajoute à cette appartenance aux mêmes organisations ou aux mêmes réseaux le fait de partager un système commun de représentations, de valeurs et de croyances (Rallet et Torre, 2004 ; Torre et Beuret, 2012). Nous verrons, là aussi, que les acteurs œuvrant sur le site d'orpaillage partagent quelques règles de base non écrites qui garantissent le fonctionnement du site et un minimum de cohésion pour la communauté.

Les proximités géographique et organisée sont reliées. D'une part, la proximité géographique peut être efficacement valorisée grâce aux relations tissées dans le cadre de la proximité organisée (Torre, 2004). D'autre part, il existe des configurations variées dans lesquelles les proximités peuvent se compenser. Ainsi, dans certaines situations territoriales de conflictualité (par exemple dans les situations où la proximité géographique est subie parce que source d'effets externes négatifs pour certains acteurs), la construction de proximités organisées est un outil de gouvernance permettant de rapprocher les acteurs (Torre et Beuret, 2012).

Plusieurs auteurs de ce courant utilisent par ailleurs une typologie permettant de distinguer les notions d'actif et de ressource et de les qualifier selon qu'ils sont génériques ou spécifiques (Colletis et Pecqueur, 1993). Dans cette perspective, les actifs sont tangibles, ils existent et sont "activés", alors que les ressources représentent des potentiels. Lorsque ces actifs ou ressources sont génériques, leur valeur n'est pas liée aux caractéristiques du territoire ou à ses composantes (économiques, humaines, sociales...). Ils ne permettent donc pas à un territoire de se distinguer et leur prix est celui du marché. En revanche, lorsque ces actifs ou ressources sont spécifiques, ils sont le fruit d'une combinaison particulière de relations, de connaissances, de compétences locales qui permettent à un territoire de se différencier.

Comme matière première, l'or est une ressource générique par excellence. Il devient un actif dès lors qu'il est extrait. Lorsqu'il est simplement vendu sur les marchés, on peut postuler qu'il reste un actif générique qui ne le distingue pas de l'or produit n'importe où ailleurs. En revanche, la configuration productive (organisation du site, 
compétences de la main-d'œuvre) peut résulter d'une histoire et de règles particulières créées dans le cadre de proximités géographiques et relationnelles. Notre hypothèse est que l'orpaillage artisanal relève bien de l'activation d'une ressource générique, mais que les modes de coordination, qui peuvent varier d'un site à l'autre selon diverses modalités, peuvent générer du développement territorial de façon différenciée selon les lieux d'extraction.

Après un bref retour sur l'organisation institutionnelle du secteur aurifère au Burkina Faso, nous présenterons le site choisi et la méthode d'enquête adoptée. Nous détaillerons ensuite le fonctionnement du site d'orpaillage étudié et les différentes parties prenantes contribuant à sa gouvernance, avant de discuter des implications sociales et économiques de cette gouvernance de proximité qui repose à la fois sur le marché et sur les structures de pouvoir coutumières.

\section{L'organisation de l'orpaillage au Burkina Faso}

L'exploitation minière au Burkina Faso est placée sous une double tutelle. Celle du ministère des Mines d'abord, qui est chargé de l'organisation, de la prospection, de la délivrance des permis de recherche et d'exploitation, ainsi que de la supervision et de la régulation des pratiques d'exploitation; celle du ministère de l'Économie et des Finances ensuite, qui assure la tutelle financière (MME, 2013). Le boom minier qui a fait du Burkina Faso le quatrième producteur d'or en Afrique de l'Ouest, depuis 2009, et la prolifération des sites d'orpaillage qui s'en est suivie ont engendré des difficultés (Antil, 2014). Parmi ces difficultés, on peut retenir la faible présence de l'État sur les sites aurifères: insuffisance de contrôles et de suivis des sites d'orpaillage, insuffisance de ressources humaines qualifiées, insuffisance de moyens matériels et logistiques et enfin, insuffisance d'encadrement et d'appui aux orpailleurs (Gueye, 2001 ; Sangaré, 2016). Cette situation a entraîné une prolifération des sites aurifères artisanaux illégaux, la non-maîtrise de la situation exacte des activités d'orpaillage et une faible implication des pouvoirs locaux (communes, populations locales) dans la gouvernance minière, en plus de conduire au développement de la fraude et de l'incivisme fiscal (Andriamasinoro et Angel, 2012 ; CES, 2012).

Face à cette nouvelle donne, le Burkina Faso a procédé en 2015 à la refonte de son code minier de 2003 (Hubert et Kupper, 2015), devenu inadapté. Rédigé dans un contexte où les communes et les régions n'existaient pas (Nyame et Blocher, 2010), ce code ne prenait pas en compte ces nouveaux acteurs de la gouvernance locale devenus incontournables. Par conséquent, certains exploitants miniers négociaient directement avec l'État central sans aucune considération pour les populations locales, quand bien même les ressources sont extraites de leur territoire (Thune, 2011). Celles-ci avaient donc l'impression d'être les grandes oubliées dans le partage de leurs ressources naturelles. Pour remédier à la situation et permettre aux populations locales de 
reprendre le contrôle de leurs ressources naturelles, des initiatives se sont développées localement, en marge des circuits administratifs habituels, pour l'exploitation artisanale des sites d'or (Sangaré, 2016). Cette exploitation, qui se fait généralement à la suite d'une entente verbale entre les populations locales et des artisans miniers, est régie par une gouvernance informelle analysée plus loin.

Les autorisations d'exploitation sont délivrées exclusivement par le ministère des Mines, plus précisément par la Direction générale des mines, de la géologie et des carrières (Luning, 2008 ; Arnaldi di Balme et Lanzano, 2014). La perception des taxes s'opère au niveau local (sur les différents sites d'orpaillage implantés sur le territoire communal) par des collecteurs communaux. Ceux-ci travaillent pour le compte de la Direction régionale des impôts, qui est une entité du ministère de l'Économie et des Finances. La collecte des taxes se fait auprès de tous ceux qui exercent une quelconque activité sur le site, que l'activité soit directement liée à l'extraction ou non et que le site soit légal ou illégal. Cela dit, s'il est vrai que la commune participe à la collecte des taxes, elle n'est toutefois pas impliquée dans les décisions d'octroi des autorisations $\mathrm{d}$ 'exploitation. Cela concerne également les populations locales, dont l'avis n'est pas pris en compte. Face à cette situation, et pour tirer leur épingle du jeu, les orpailleurs ont jugé nécessaire de s'organiser aussi bien sur le plan stratégique, à travers la création d'une organisation faîtière, que sur le plan opérationnel, à travers le découpage spatial de leur espace de travail.

La loi définit l'exploitation artisanale traditionnelle comme toute « opération qui consiste à extraire et concentrer des substances minérales et à en récupérer les produits marchands pour en disposer en utilisant des méthodes et procédés traditionnels et manuels $[\ldots]^{1} »$. Au niveau structurel, il existe une organisation faîtière des orpailleurs artisanaux : le Syndicat national des orpailleurs artisanaux et traditionnels du Burkina (SY.N.OR.AR.TRA.B). Créé en 2008, il compte plusieurs centaines d'orpailleurs répartis sur l'ensemble du territoire national. Cette organisation a pour mission de défendre les intérêts moraux, matériels, économiques et professionnels des orpailleurs du Burkina $\mathrm{Faso}^{2}$. Le syndicat agit de nos jours comme interface entre les orpailleurs, les communautés, les compagnies minières et l'État en cas de conflit. Le syndicat mène périodiquement des séances de sensibilisation et de formation de ses membres sur les méfaits de la drogue ainsi que sur l'effet du cyanure et du mercure sur l'environnement et la santé humaine et animale.

\footnotetext{
${ }^{1}$ Code Minier 2003 : http://www.droit-afrique.com/upload/doc/burkina/Burkina-Code-minier2009.pdf.

${ }^{2}$ Récépissé de déclaration d'association n²008-523/MATD/DGLPAP/DAPCR de juillet 2008.
} 


\section{La commune de Gbomblora}

Située à une vingtaine de kilomètres de Gaoua, le chef-lieu de la région du Sud-Ouest, la commune rurale de Gbomblora fait partie des dix (10) départements de la province du Poni. Elle est délimitée au nord par le département de Bousséra, au sud par les départements de Legmoin et Midebdo dans la province du Noumbiel, à l'est par le Ghana et à l'ouest par le département de Gaoua. Elle couvre une superficie de $581 \mathrm{~km}^{2}$ (Commune de Gbomblora, 2014).

Figure 1 : Situation de la commune de Gbomblora

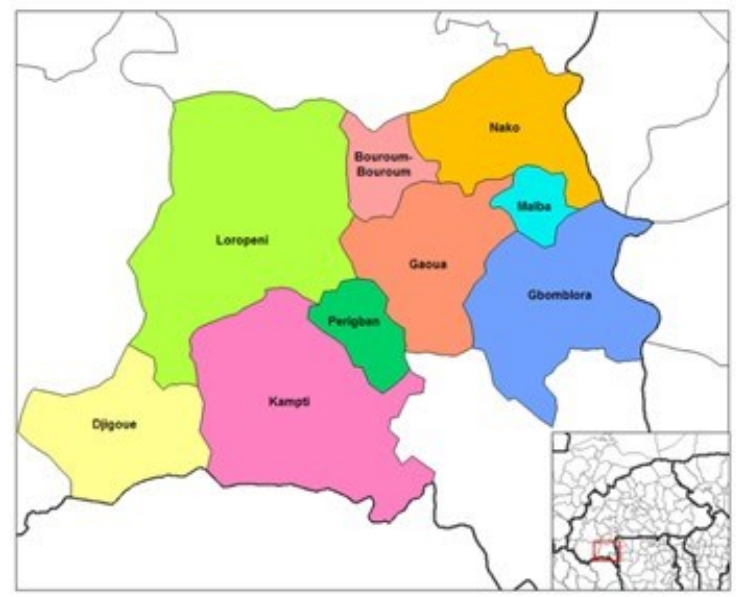

Source : Poni Departments. (s.d.). Dans Wikipédia, l'encyclopédie libre. Repéré le 3 janvier 2017 à https://en.wikipedia.org/wiki/File:Poni departments.png

Les activités socioéconomiques dominantes dans la commune sont l'agriculture, l'artisanat et l'orpaillage artisanal. En 2012, on a répertorié sur le territoire de la commune de Gbomblora six sites d'orpaillage (Bontara, Pélinka Yourbi, Tantouo, Tiémolo et Wadaradouo), tous illégaux (MATS³, 2012 ; Toé, 2013). Notre analyse porte plus précisément sur le site de Pélinka, un site créé en 2005 dans la partie nord-est du village de Gbomblora et qui constitue le plus gros site de la commune. Au moment de notre enquête au printemps 2015, il comptait plus de 200 personnes, mais cette population est extrêmement fluctuante. Le site a connu plusieurs mouvements de personnes liés au fait que les orpailleurs n'hésitent pas à plier bagage dès lors qu'ils entendent parler de l'ouverture d'un nouveau site d'orpaillage réputé plus productif ailleurs (Sangaré, 2016).

L'orpaillage se pratique depuis longtemps dans cette région, qui est considérée depuis le $14^{\text {e }}$ siècle comme une des plus grandes zones aurifères du pays (Père, 1992). À cette époque, les habitants s'adonnaient à la recherche de l'or de manière traditionnelle, c'est-à-dire en récoltant l'or manuellement et en surface. La recherche et la manipulation de l'or étaient des activités traditionnellement dévolues aux femmes,

\footnotetext{
${ }^{3}$ Ministère de l'Administration territoriale et de la Sécurité
} 
qui se contentaient de chercher l'or le long des cours d'eau à l'aide de la bâtée (Schneider, 1993; Sawadogo, 2011). L'or était ensuite vendu lorsque la famille avait un urgent besoin d'argent pour faire face à des dépenses précises, comme l'achat des vivres durant les périodes difficiles, ou aux dépenses inhérentes aux cérémonies coutumières, comme l'achat d'animaux ou de volailles pour les sacrifices rituels, l'organisation des funérailles ou les cérémonies d'initiation, par exemple (Schneider, 1993). Cette activité se pratiquait sans outillage ni substances chimiques comme le mercure et le cyanure. La pratique de l'orpaillage a évolué et s'est transformée par la suite. En fait, l'orpaillage sur une base quotidienne intensive tel que mené actuellement est une pratique récente. L'arrivée massive des orpailleurs dans la région du Sud-Ouest et l'organisation de la pratique de l'orpaillage dans sa forme actuelle datent des années 1990 (Mégret, 2008). Les populations autochtones, issues majoritairement du peuple Lobi, considèrent l'or comme une matière vivante, mais également maléfique et dangereuse. Plusieurs auteurs (Père, 1992 ; Schneider, 1993 ; Werthmann, 2003 ; Mégret, 2008) ont largement décrit le caractère sacré de l'or et les interdits entourant ce métal précieux dans cette partie du pays. Cette perception de l'or est encore largement partagée par les populations autochtones, ce qui explique qu'aucune activité extractive n'était menée comme activité principale de manière formelle et organisée avant l'arrivée d'orpailleurs d'autres régions.

Notre enquête sur le site s'est déroulée de janvier à avril 2015 (Sangaré, 2016). Outre les nombreuses observations réalisées directement sur le site, nous avons conduit des entretiens semi-directifs avec divers intervenants : les responsables villageois (le chef du village et le chef des terres), des représentants des administrations locales (maire, conseiller communal, conseiller du conseil villageois de développement) et divers services techniques décentralisés $(n=11)$, ainsi que des chefs de ménage associant de façon différenciée agriculture et orpaillage artisanal ${ }^{4}$ dans leur système d'activités (n $=25)$.

\section{Fonctionnement et gouvernance du site de Pélinka}

Avant de décrire l'organisation du site de Pélinka, il convient de clarifier le statut des orpailleurs artisanaux et leur rapport avec les autorités (administratives et coutumières). Comme nous l'avons vu dans la section 1 , le secteur aurifère est régi par un ensemble de dispositions légales au niveau national. L'obtention des autorisations d'exploitation nécessitant des démarches exigeantes, de nombreux sites sont exploités illégalement parce qu'ils n'ont pas obtenu les autorisations du ministère des Mines. Cela dit, un autre niveau de gouvernance plus informel est à considérer : celui des autorités coutumières locales qui déterminent leurs propres règles d'autorisation.

\footnotetext{
${ }^{4}$ Les ménages rencontrés ont été divisés en trois sous-groupes : ménages pratiquant essentiellement l'orpaillage $(n=12)$, ménages pratiquant l'agriculture et l'orpaillage $(n=7)$, et ménages pratiquant essentiellement l'agriculture $(n=6)$.
} 
Nous avons ainsi identifié quatre cas de figure dans l'exploitation d'un site d'orpaillage au Burkina Faso, lesquels sont présentés dans le tableau 1 ci-dessous.

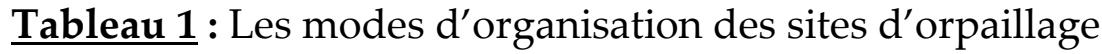

\begin{tabular}{|c|c|c|}
\hline & $\begin{array}{c}\text { Site exploité avec l'accord des } \\
\text { autorités administratives }\end{array}$ & $\begin{array}{c}\text { Site exploité sans l'accord des } \\
\text { autorités administratives }\end{array}$ \\
\hline $\begin{array}{l}\text { Site exploité avec l'accord des } \\
\text { autorités coutumières }\end{array}$ & $\begin{array}{l}\text { Les orpailleurs détiennent à la } \\
\text { fois la légitimité } \\
\text { communautaire et la légalité } \\
\text { administrative (site } \\
\text { d'orpaillage légal). L'accord } \\
\text { des autorités coutumières } \\
\text { confère aux orpailleurs la } \\
\text { légitimité communautaire. Les } \\
\text { deux sont indépendantes. }\end{array}$ & $\begin{array}{l}\text { Les orpailleurs se contentent } \\
\text { d'obtenir l'accord des autorités } \\
\text { coutumières et exploitent le } \\
\text { site sans autorisation écrite du } \\
\text { ministère des Mines (site } \\
\text { d'orpaillage illégal). Le site de } \\
\text { Pélinka correspond à ce cas de } \\
\text { figure. }\end{array}$ \\
\hline $\begin{array}{l}\text { Site exploité sans l'accord des } \\
\text { autorités coutumières }\end{array}$ & $\begin{array}{l}\text { Les orpailleurs détiennent un } \\
\text { permis délivré par le ministère } \\
\text { des Mines, mais n'ont pas } \\
\text { sollicité l'accord des autorités } \\
\text { coutumières même si certains } \\
\text { d'entre eux essaient de susciter } \\
\text { la sympathie de la } \\
\text { communauté locale afin } \\
\text { d'éviter les conflits avec les } \\
\text { populations locales. Il s'agit } \\
\text { généralement de sites exploités } \\
\text { par des sociétés minières } \\
\text { industrielles. }\end{array}$ & $\begin{array}{l}\text { Les orpailleurs n'ont ni la } \\
\text { légitimité communautaire ni le } \\
\text { permis d'exploiter délivré par } \\
\text { le ministère des Mines. Ils } \\
\text { exploitent une parcelle en } \\
\text { brousse sans l'accord de la } \\
\text { communauté à qui } \\
\text { appartiennent ces terres. Ce cas } \\
\text { de figure est généralement à } \\
\text { l'origine des conflits entre } \\
\text { orpailleurs et autochtones. }\end{array}$ \\
\hline
\end{tabular}

À Gbomblora, la gouvernance coutumière au niveau du village est assurée par un collège de sages à la tête duquel il y a le chef du village et le chef des terres. En termes $\mathrm{d}$ 'attributions, le chef de village assure la gestion morale et politique du village, tandis que le chef des terres est responsable de la dimension spirituelle de la gestion de la communauté. Il est considéré comme l'intermédiaire entre la communauté et les divinités. À ce titre, il fait office de prêtre garant des forces occultes et des fétiches du village, et est chargé de diriger les cérémonies rituelles réparatrices en cas de violation des normes sociales du village. C'est le cas par exemple de la profanation des lieux sacrés (forêts sacrées, tombes, etc.) par certains orpailleurs. Il est également responsable de la gestion des problèmes fonciers.

Lorsqu'un nouvel orpailleur arrive en quête d'une parcelle d'exploration dans le village ou des environs, il est d'abord reçu par le propriétaire de la parcelle convoitée (propriétaire terrien). Ce dernier entame une discussion avec l'arrivant pour connaître ses motivations et les conditions dans lesquelles il envisage de mener ses activités. À la suite de cette entrevue, les deux parties s'entendent sur la nature des droits et la 
somme à payer au propriétaire terrien. Les frais engagés par le demandeur auprès du propriétaire terrien peuvent varier d'un propriétaire à l'autre. La transaction se fait sur la base d'un contrat verbal.

Une fois que les deux parties tombent d'accord, le propriétaire terrien conduit l'orpailleur auprès $\mathrm{du}$ « chef de terre » qui lui indique ce qu'il devra apporter en guise d'offrandes (poulets, boucs, cola, etc.) à sacrifier aux divinités sur l'autel. Cette cérémonie se fait en présence des notables du village et des familles concernées. C'est seulement une fois qu'il a satisfait à ces exigences que l'orpailleur est autorisé à commencer ses activités extractives. Cette autorisation lui confère, de fait, la légitimité communautaire et le statut de responsable du site. On remarque ainsi l'activation de la proximité organisée par les échanges directs entre les protagonistes d'une part (logique d'appartenance), et par une conception partagée de ce qui fonde le contrat verbal passé entre eux d'autre part (logique de similitude).

À partir de ce moment, tout nouvel orpailleur qui arrive sur le site doit passer par le responsable de site pour s'acquitter des frais avant d'être autorisé à exercer. Une partie de ces frais est reversée au propriétaire terrien, qui passe périodiquement pour vérifier le nombre de trous creusés ainsi que le nombre de hangars dressés sur le site.

Le responsable de site est généralement un commerçant ou une personne qui possède les moyens financiers nécessaires à la mise de fonds initiale. C'est à lui, en tant que répondant du site vis-à-vis des autorités coutumières et du propriétaire terrien, que s'adressent toutes les personnes souhaitant exercer une activité sur le site. On parle ici des creuseurs, bien entendu, mais aussi de tous les autres acteurs qui devront lui payer un droit d'accès au site: broyeurs, concasseurs, laveurs, restaurateurs, mécaniciens, coiffeurs, prostituées, etc. Ce droit d'accès peut être payé en nature, dans le cas des propriétaires de trou, ou en espèces en ce qui concerne les autres acteurs. Pour ces derniers, la somme à payer varie en fonction du nombre de « constructions » installées sur le site.

Les personnes exerçant des activités sur le site relèvent de diverses catégories :

- Les propriétaires de trou. Ceux-ci ont acquis le droit de creuser un trou auprès $\mathrm{du}$ responsable $\mathrm{du}$ site. Ils ne creusent pas directement, mais font travailler des creuseurs sur la base d'une clé de répartition des minerais extraits (50-50 selon nos observations). Plus le nombre de trous qu'ils possèdent est élevé, plus leurs gains sont importants.

- Les creuseurs. Ceux-ci travaillent dans les trous. Ils se partagent les minerais avec le propriétaire du trou. Ils fonctionnent selon un mode rotatif qui fait que dans chaque trou, il existe au moins deux groupes de creuseurs : un groupe travaille toute la journée et le second prend le relais pour travailler de la tombée de la nuit au lever du jour.

- $\quad$ Les acteurs chargés du traitement du minerai. Il s'agit des broyeurs, des concasseurs, des laveurs, des dynamiteurs et des personnes chargées de l'amalgamation. Ce sont des prestataires autonomes qui travaillent sur le 
site et qui sont payés à l'acte par les détenteurs de minerais qui sollicitent leurs services. Certains possèdent des machines (concasseurs).

- Les acteurs chargés de l'achat et la vente de l'or. Installés sur le site, ils achètent l'or produit sur le site pour le compte du responsable du site. Ils n'ont pas affaire au propriétaire terrien et n'ont de comptes à rendre qu'au responsable du site pour qui ils travaillent.

- Les acteurs engagés dans des activités annexes. Ceux-ci ne sont concernés ni par l'extraction ni par le traitement ou la commercialisation de l'or. Ils profitent de la présence du site et des orpailleurs pour faire des affaires et conduire diverses activités génératrices de revenus sur le site d'orpaillage (restauration, coiffure, commerce, mécanique, boucherie, porteurs d'eau, prostitution...). Eux aussi paient annuellement au responsable du site un droit d'usage pour l'espace qu'ils occupent sur le site.

La répartition des revenus entre les divers acteurs concernés suit la hiérarchie des métiers. Dans le tableau 2, nous présentons les revenus mensuels moyens rapportés par les différents acteurs rencontrés.

Tableau 2 : Revenu mensuel moyen par personne et par type d'emploi sur le site d'orpaillage

\begin{tabular}{lcc}
\hline \multicolumn{1}{c}{ Emplois } & $\begin{array}{c}\text { Revenu mensuel moyen/tête } \\
\text { (FCFA) }\end{array}$ & $\begin{array}{c}\text { Valeur en dollars } \\
\text { canadiens }\end{array}$ \\
\hline $\begin{array}{l}\text { Responsable du site } \\
\text { Propriétaires de } \\
\text { puits }\end{array}$ & + de 6000000 & 12000 \\
Creuseurs de puits & $125000-6000000$ & $250-12000$ \\
Broyeurs & $10000-500000$ & $20-1000$ \\
Concasseurs & $20000-125000$ & $40-250$ \\
Laveurs & $20000-250000$ & $40-500$ \\
Dynamiteurs & $15000-225000$ & $30-450$ \\
\hline
\end{tabular}

Source : enquêtes de terrain (Sangaré, 2016).

En résumé, le responsable du site coordonne les activités et fixe les règles, il ne creuse pas de trou et ne mène aucune activité manuelle. C'est un homme d'affaires ou un commerçant fortuné dont le rôle consiste à identifier un site propice, à mener les démarches auprès des propriétaires terriens et des autorités coutumières et à conclure avec eux les modalités financières de l'exploitation du site. Pour le reste, ce sont les chercheurs d'or qui viennent vers lui pour négocier leur installation. Il peut se faire représenter au quotidien par une personne de confiance et passer de temps en temps superviser et encaisser l'argent versé par les nouveaux arrivants. 
De manière générale, en dehors des conditions financières et coutumières à respecter pour être accepté sur le site, trois infractions peuvent conduire à l'expulsion d'un acteur : le viol, l'adultère et le vol. Contrairement aux conditions financières qui sont négociables, ces trois actes sont considérés comme des délits très graves, et l'expulsion n'est alors pas négociable. Au-delà de la dimension morale condamnable, ces actes sont considérés par les orpailleurs comme portant malheur et pouvant rendre le site aurifère improductif.

\section{L'organisation spatiale du site de Pélinka}

Sur le plan opérationnel, le site d'orpaillage de Pélinka est délimité en trois zones distinctes d'activités que sont la zone de creusage, la zone de traitement et la zone d'habitation. Ainsi, la proximité géographique est structurée en trois espaces fonctionnels qui ont chacun leur vocation spécifique et sont situés à quelques kilomètres de distance les uns des autres.

La figure 2 ci-dessous représente le découpage instauré par les orpailleurs pour séparer physiquement les trois zones. Malgré cette séparation, le développement des activités entraîne un débordement et un rapprochement entre la zone d'habitation et la zone de traitement. Cela est lié au développement et à l'expansion des abris construits régulièrement en fonction des besoins et de l'arrivée de nouveaux acteurs. De plus, il arrive que les orpailleurs apportent des minerais de la zone de creusage pour les traiter sur le site d'habitation.

\section{Figure 2 : 1'organisation du site d'orpaillage en 3 zones spécifiques}

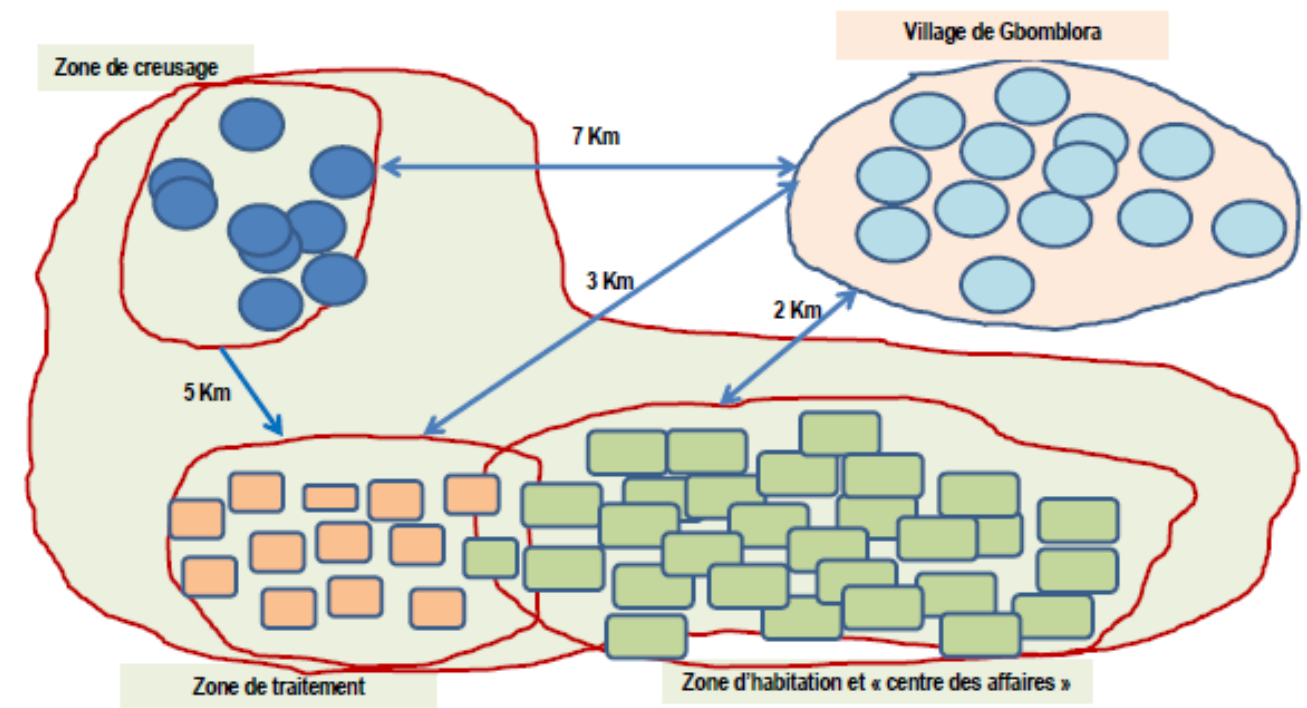

Source : enquêtes de terrain (Sangaré, 2016). 
La zone de creusage est réservée au creusage des trous et galeries en vue de l'exploitation des gisements. Cette zone est parsemée de trous et de gravats de toutes sortes disséminés à perte de vue. Le terrain est jonché de tas de roches sorties du soussol. Une fois les minéraux sortis de terre, des orpailleurs s'activent pour les conditionner dans des sacs de $50 \mathrm{~kg}$ en vue de leur évacuation vers la zone de traitement.

Sur la zone de traitement sont installés les différents acteurs assurant le broyage, le concassage, le lavage, l'amalgamation et l'achat de l'or. Ces différents acteurs sont installés sous des hangars disséminés de part et d'autre de la parcelle. Ils reçoivent et traitent les minerais provenant du site de creusage moyennant des tarifs bien précis. Les concasseurs, les propriétaires de moulins (broyeurs) et les amalgameurs sont en général des prestataires qui ne sont pas propriétaires des minerais qu'ils traitent. Les unités de mesure des quantités d'or extraites sont fixées de façon conventionnelle selon les équivalences présentées dans le tableau 3 ci-dessous.

$\underline{\text { Tableau } 3}$ : Unités de mesure et valeurs correspondantes

\begin{tabular}{lc}
\hline \multicolumn{1}{c}{ Unité de mesure } & Poids $(\mathrm{g})$ \\
\hline $\mathbf{1}$ pièce de 1 franc CFA & $1 \mathrm{~g}$ \\
$\mathbf{1}$ pièce de 10 francs CFA & $3 \mathrm{~g}$ \\
$\mathbf{1}$ pièce de 25 francs CFA & $6 \mathrm{~g}$ \\
$\mathbf{1 2}$ brins d'allumette & $1 \mathrm{~g}$ \\
$\mathbf{1}$ brin d'allumette & $0,083 \mathrm{~g}$ \\
\hline
\end{tabular}

Source : enquêtes de terrain (Sangaré, 2016).

Selon la loi, l'or transformé sur le site devrait être vendu aux acheteurs agréés par l'État. En réalité, l'or est acheté aussi bien par des personnes représentant des comptoirs agréés que par des acheteurs indépendants. Lorsque les orpailleurs estiment que les prix proposés au niveau local sont trop bas, ils se rendent de l'autre côté de la frontière (Ghana, Côte d'Ivoire) située à quelques kilomètres du site pour vendre l'or à un meilleur prix aux acheteurs installés dans ces deux pays. La proximité géographique, la porosité des frontières et la faiblesse de la surveillance facilitent cette pratique courante qui constitue une perte pour le Trésor public. Ces opérations ne sont pas déclarées, surtout lorsqu'il s'agit de sites clandestins et illégaux. Selon le ministère des Mines, $90 \%$ de la quantité d'or extraite artisanalement n'est pas déclarée. Il est également important de signaler à ce sujet que sur les 84 sites répertoriés en 2012 dans la région du Sud-Ouest, seuls 17 sont officiels et disposent d'une autorisation officielle d'exploitation (MATS, 2012).

La zone d'habitation du site d'orpaillage de Pélinka est à la fois l'espace de vie et le lieu où se déroulent toutes les activités annexes. Cette zone qui fait également office de «centre des affaires » occupe une superficie d'environ cinq hectares. On y trouve 
une multitude de hangars de paille ainsi que des baraquements en tôles ondulées. Ces installations abritent aussi bien des habitations que des commerces de toutes sortes: salons de coiffure, restaurants, grilleurs de brochettes de viande, vendeurs d'essence, ateliers de soudure et ateliers de mécanique pour la réparation des motocyclettes, des moulins ou des groupes électrogènes. On y trouve également des vendeurs de médicaments communément appelés pharmacie par terre. Ces médicaments sont vendus par de jeunes gens qui ne savent ni lire ni écrire, mais qui «conseillent» les clients sur les vertus des différents produits qu'ils vendent. Ces produits pharmaceutiques, généralement périmés, sont étalés au soleil, à proximité d'autres marchandises telles que chaussures, outils, etc. Dans le tas de médicaments, on trouve des antalgiques, des antibiotiques, des amphétamines, des aphrodisiaques, etc. Ces médicaments proviennent des pays voisins et semblent être introduits frauduleusement. En dépit des risques qu'ils représentent, ces médicaments sont très prisés des orpailleurs en raison non seulement de leur coût abordable, mais aussi de la proximité géographique. Car contrairement aux centres de santé officiels, ces vendeurs ambulants vont vers les orpailleurs, qui n'ont plus besoin de suspendre leurs activités pour se rendre au dispensaire. Sur le site, se trouvent également des vendeurs de médicaments traditionnels (faits de poudres, de décoctions) censés apporter protection et chance dans la recherche de l'or. Certains sont utilisés aussi pour guérir différents maux.

Il règne sur la zone d'habitation un vacarme constant de bruits de machines, de motocyclettes en réparation et de musique distillée par les haut-parleurs des débits de boisson ainsi que des vidéoclubs. La zone est animée 24 heures sur 24 , ce qui fait que les commerces demeurent ouverts toute la nuit pour satisfaire les besoins des orpailleurs (creuseurs, broyeurs, etc.). Il existe également une certaine solidarité non seulement entre les orpailleurs, mais également entre orpailleurs et marchands. Il n'est donc pas rare de voir les nouveaux arrivants bénéficier d'un soutien des plus anciens, sous forme de prêts en argent ou en nature (outils, vêtements, médicaments, nourriture, etc.), pour faciliter le démarrage de leur activité. Ces prêts sont remboursables dès que la situation financière de l'intéressé le permet. Cette forme d'entraide informelle comble le vide laissé par l'absence d'institutions de microcrédit ou d'assurance. Selon le responsable du site, il est arrivé que les orpailleurs organisent des cotisations volontaires pour assurer la prise en charge des frais médicaux ou du transport d'un orpailleur malade ou décédé vers son village, ou le déménagement de la veuve et des orphelins des orpailleurs dans leur village d'origine. Lorsqu'un décès survient, les orpailleurs s'organisent toujours pour qu'un groupe de volontaires accompagne la dépouille ou la veuve et les orphelins jusqu'à destination, participe aux funérailles et présente les condoléances à la famille du défunt au nom de ses camarades du site. Cela est extrêmement important dans un contexte où il n'existe ni assurance sociale ni système de retraite formel, et encore moins de possibilités de prêts bancaires en faveur des orpailleurs. C'est ici que la proximité organisée prend tout son sens : face au vide institutionnel étatique existant à ce niveau, les orpailleurs ont mis 
en place un système informel de prise en charge des cas sociaux exclusivement basé sur la cotisation volontaire des acteurs travaillant sur le site.

La zone d'habitation abrite également des lieux de loisirs comme des clubs vidéo dans lesquels les orpailleurs et autres acteurs peuvent visionner des films ou des matchs de football moyennant le paiement d'un droit d'entrée de 200 FCFA (0,43 dollar canadien) par séance. Ces clubs vidéo, tous dotés d'antennes paraboliques, sont alimentés en énergie par des groupes électrogènes ou, pour certains, par des panneaux solaires. Il existe aussi plusieurs débits de boissons disséminés parmi les habitations et où on peut se procurer des boissons alcoolisées et non alcoolisées.

Enfin, dans un coin de la zone d'habitation se trouvent les maisons abritant des femmes qui s'adonnent à la prostitution. Ces femmes louent des « chambres » auprès d'un tenancier de bar à raison de 1000 FCFA (2 dollars canadiens) par jour et par fille pour toute la durée de leur séjour. Le tenancier du bar (propriétaire des « chambres ») paie de son côté au responsable du site un droit sur le prix de la location de ses « chambres » (25000 FCFA, soit 54 dollars canadiens) par « chambre » et par an.

Les habitats précaires sont occupés aussi bien par des orpailleurs que par les personnes exerçant des activités annexes de prestation de services (bouchers, mécaniciens, vendeurs de cordes, de sacs, etc.) sans liens directs avec le travail d'extraction. Cela dit, certains orpailleurs, bien que possédant un baraquement sur le site, préfèrent aller habiter au village et effectuer la navette chaque jour entre le village et le site distant d'environ $2 \mathrm{~km}$. Dans l'autre sens, les habitants du village trouvent sur le site des services et des marchandises qui n'existent pas au village. La proximité géographique entre le site et le village, subie dans un premier temps par les villageois, a ensuite été activée, d'une part parce qu'elle permet aux villageois de développer des activités leur procurant des revenus, et d'autre part parce qu'elle leur permet de s'approvisionner en tout temps en divers produits. En effet, Gbomblora ne dispose pas de marché et les habitants doivent se rendre sur les marchés des villages voisins, dont les plus proches sont Doudou $(10 \mathrm{~km})$, Gaoua $(20 \mathrm{~km})$ et Tobo $(32 \mathrm{~km})$. Mentionnons en particulier le cas de la viande, qui est disponible en permanence sur le site d'orpaillage, alors qu'il n'y a pas d'élevage à Gbomblora ${ }^{5}$. Ainsi, le site d'orpaillage est devenu le centre économique et le cœur de l'économie du village, et le seul lieu de Gbomblora où les habitants peuvent se procurer en permanence divers services ou marchandises.

\section{Conclusion}

Contrairement aux préjugés selon lesquels les sites d'orpaillage sont des espaces de non-droit, l'étude du site de Gbomblora a permis de découvrir que derrière un

\footnotetext{
${ }^{5}$ Contrairement aux autres localités de la province, Gbomblora n'est pas une zone d'élevage.
} 
apparent désordre se cache en réalité un ordre complexe, reposant sur une gouvernance de proximité articulée aux structures de chefferie coutumières. La proximité géographique entre villageois autochtones et orpailleurs, subie dans un premier temps, a rapidement été activée par les échanges économiques entre le village et le site d'orpaillage. La proximité organisée se décline pour sa part au travers de ses deux logiques : l'appartenance, dans la mesure où tous les acteurs appartiennent au fond à la même organisation même si celle-ci reste informelle, et la similitude, dans la mesure où les règles et les interdits font l'objet d'un consensus implicite qui assure la cohésion sur le site.

Les règles régissant le fonctionnement du site de Pélinka ne sont pas dictées par l'État, pratiquement absent du site, mais découlent plutôt d'une gouvernance résultant de l'activation des proximités géographique et organisée, ce que nous appelons ici gouvernance de proximité. Elles sont à la fois locales et informelles, dans le sens où tous les accords, toutes les transactions, reposent sur des contrats oraux et des conventions locales, comme nous l'avons vu par exemple à propos des unités de mesure. Cette gouvernance locale est extrêmement hiérarchisée, partant du trio constitué par le propriétaire terrien, le responsable de site et le chef de terre, jusqu'aux creuseurs. Il revient à tous les acteurs de se conformer aux règles édictées par ce trio ou de quitter les lieux. Le responsable du site et le propriétaire terrien se partagent une part importante de la rente, mais notre enquête a aussi montré que l'orpaillage artisanal apporte un complément de revenu indispensable à des ménages ruraux pauvres pour qui il est très difficile de vivre uniquement de l'agriculture. Pour une partie de ces orpailleurs, les revenus issus de l'orpaillage permettent même de consolider l'exploitation agricole.

D'un point de vue social, les règles mises en œuvre, même si elles sont insuffisantes, garantissent tout de même la régulation de l'activité et offrent une forme d'équilibre et de cohésion qui permet la plupart du temps d'éviter les conflits, notamment entre orpailleurs allochtones et autochtones. Cela ne fait pas pour autant de l'or extrait sur le site un actif spécifique. D'une part, l'or n'est pas transformé sur le site. Nous n'avons constaté aucune innovation sociale ou technologique permettant au site de se distinguer des autres sites du point de vue des produits commercialisés. D'autre part, une portion importante de la rente est captée par le responsable du site et le propriétaire terrien, contrairement aux formes de gouvernance plus communautaires et collectives observées sur d'autres sites, notamment au Mali (Arnaldi di Balme et Lanzano, 2014; Teschner, 20146). Cela dit, par l'activité qu'il procure pour de

\footnotetext{
${ }^{6}$ Dans le sud du Mali, il existe un système de gouvernance et de propriété traditionnelle caractérisé par une association d'orpaillage nommée « tomboloma » supervisée par le chef du village. Le rôle de l'association est de gérer, de surveiller, de régler les conflits et de collecter les taxes au nom du chef de village et des chefs de terres dans les camps miniers présents sur son territoire. C'est également cette association qui distribue les terres aux personnes souhaitant entreprendre des activités d'extraction artisanale. Ainsi, dans l'étude réalisée par Arnaldi di Balme \& Lanzano (2014) sur les sites
} 
nombreux ménages, par les revenus qu'il génère et par les opportunités qu'il offre aux villageois de Gbomblora de vendre leurs produits ou services et de pouvoir s'approvisionner localement, le site contribue au développement du village.

Toutefois, cette organisation demeure fragile et comporte des insuffisances qui limitent la contribution de l'orpaillage au développement territorial. L'absence de reconnaissance formelle $\mathrm{du}$ site d'orpaillage par l'administration contribue au maintien des pratiques préjudiciables du point de vue environnemental, sanitaire, social et même fiscal. Elle empêche également les acteurs d'avoir accès à certains programmes gouvernementaux (formation, organisation, équipement, appui technique). S'il est vrai que l'orpaillage occupe de plus en plus une place centrale dans l'économie de la commune rurale de Gbomblora, son mode d'exploitation reste principalement orienté vers la rentabilité à court terme, ce qui rend le travail dangereux et précaire, et fait obstacle à la durabilité sociale et environnementale.

Ainsi, cette gouvernance de proximité qui repose à la fois sur le marché et sur les structures de pouvoir coutumières, souffre de ne pas être suffisamment articulée à une politique publique structurante, accompagnant et respectant tout à la fois les mécanismes locaux de régulation.

\section{Bibliographie}

Airault, P. (2012). Burkina Faso : la soif de l'or. Jeune Afrique, http://www.jeuneafrique.com/Articles/Dossier/JA2668p085.xml0/or-investissementmines-gouvernanceburkina-faso-la-soif-de-l-or.htmlContexte

Andriamasinoro, F. et Angel, J. M. (2012). « Artisanal and small-scale gold mining in Burkina Faso: suggestion of multi-agent methodology as a complementary support in elaborating a policy ». Resources Policy, 37(3) : 385-396.

Antil, A. (2014). The Mining Boom in the Sahel Region: Will the Development last? Paris : Institut français des relations internationales.

Arnaldi di Balme, L. et Lanzano, C. (2014). «Gouverner l'éphémère. Étude sur l'organisation technique et politique de deux sites d'orpaillage (Bantara et Gombélèdougou, Burkina Faso) », Étude Récit n 37. Ouagadougou : Laboratoire Citoyennetés.

d'extraction artisanale de Bantara et Gombélèdougou, il existe une gouvernance structurée calquée sur les lois formelles, dirigée par un comité villageois, des autorités coutumières et des migrants. Dans la commune de Yallankoro-Soloba étudiée par Teschner (2014), l'auteur montre comment la commune a pu financer ses dépenses de fonctionnement (telles que les paies des enseignants des écoles), par l'instauration de diverses taxes sur les activités d'orpaillage. Cela a permis selon l'auteur de faire face au retrait des partenaires du Mali (Union européenne, Banque mondiale,...) suite à la crise politique survenue au Mali en 2012. 
Banchirigah S. (2006). « How reforms have fuelled the expansion of artisanal mining? Evidence from sub-saharan Africa », Resources Policy 31(3) : 165-171.

Beaurain C., Longuépée J., et Pannekoucke Soussi S. (2009). «La proximité institutionnelle, condition à la reconquête de la qualité de l'environnement. L'exemple de l'agglomération dunkerquoise », Natures Sciences Sociétés, 17 (4) : 373 380

Bellet M., Colletis G., et Lung Y. (dir.) (1993). «Économie de proximités », Revue d'économie régionale et urbaine, (3) : 357-361.

Bouba-Olga, O., Coris, M., et Carrincazeaux, C. (2008). «Avant-propos ». Revue d'Économie régionale et urbaine, (3) : 279-287.

Carrincazeaux C., Lung Y., et Vicente J. (2008). « The Scientific Trajectory of the French School of Proximity: Interaction- and Institution-based Approaches to Regional Innovation Systems », European Planning Studies, 16(5) : 617-628.

CES. (2012). Expansion du secteur minier et développement durable au Burkina Faso : cas de l'exploitation aurifère, $75 \mathrm{p}$.

Colletis G., et Pecqueur B. (1993). «Intégration des espaces et quasi-intégration des firmes : vers de nouvelles rencontres productives ? ", Revue d'économie régionale et urbaine, (3) : 489-508.

Commune de Gbomblora. (2014). Plan communal de développement 2015-2019 de la commune rurale de Gbomblora.

Doucouré, B. (2014). «Développement de l'orpaillage et mutations dans les villages aurifères du sud-est du Sénégal », Africa Development, 39 (2) : 47-67.

Filippi M., et Torre A. (2003). « Local organization and institutions. How can geographical proximity be activated by collective projects? », International Journal of Technology Management, 26 (2-4) : 386-400.

Gilly J.-P. et Lung Y. (2005). " Proximités, secteurs et territoires », Cahiers du Grès, (09), 20 p.

Gilly J. P., et Pecqueur B. (2000). « Régulation des territoires et dynamiques institutionnelles de proximité : le cas de Toulouse et des Baronnies » In Gilly J. P., Torre A. (dir.), Dynamiques de proximité. L'Harmattan : Paris : 131-164.

Gilly J.-P., et Torre A. (2000). Dynamiques de proximité, 1'Harmattan : Paris.

Gueye, D. (2001). Small-scale mining in Burkina Faso. England : International Institute for Environment and Development.

Gylfason, T. et Zoega, G. (2006). « Natural resources and economic growth: The role of investment ». The World Economy, 29(8) : 1091-1115.Hentschel T., Hruschka F., et Priester M. (2002). Global Report on Artisanal et Small-Scale Mining. Mining, Minerals and Sustainable Development $n^{\circ} 70$. 
Hilson, G. (2002). « Land Use Competition Between Small and Large Scale Miners: a Case Study of Ghana ». Land Use Policy, 19(2) : 149-156.

Hilson, G., et Garforth, C. (2013). « Everyone is Now Concentrating on the Mining: Drivers and Implications of Rural Economic Transition in the Eastern Region of Ghana ». Journal of Development Studies, 49(3) : 348-364.

Hubert, N. et Kupper, C. (2015). Nouveau code minier au Burkina: de l'or au bénéfice de tous? $12 \mathrm{p}$.

Luning, S. (2008). « Liberalisation of the Gold Mining Sector in Burkina ». Review of African Political Economy, 35 (117) : 387-401.

Maradan, D., Ouédraogo B., Thiombiano N., Thiombiano T., et Zein K. (2011).

Analyse économique du secteur des mines. Liens pauvreté et environnement. Burkina Faso, ministère de l'Environnement et du Cadre de Vie (MECV). Rapport final, 69 p.

MATS. (2012). Session extraordinaire du cadre de concertation régional sur l'exploitation artisanale traditionnelle de l'or dans la région du Sud-ouest. 75 p.

Mégret Q. (2008), «L'or mort ou vif : L'orpaillage en pays lobi burkinabé », in M. Croset J. Bonhomme, Déjouer la mort en Afrique - Or, orphelins, fantômes, trophées et fétiches, Paris : L'Harmattan : 15-41.

MME. (2013). Politique sectorielle des mines 2014 - 2025. Rapport, 66 p.

Nyame, F., et Blocher, J. (2010). «Influence of land tenure practises on artisanal mining activity in Ghana ». Resources Policy, (35) : 47-53.

Okoh, G., et Hilson, G. (2011). « Poverty and livelihood Diversification: Exploring the Linkages Between Smallholder Farming and Artisanal Mining ». Journal of International Development, 23(8) : 1100-1114.

OMS. (2013). Prévenir la maladie grâce à un environnement sain : l'exposition au mercure et ses conséquences sanitaires chez les membres de la communauté minière artisanale et à petite échelle de l'or (ASGM). 7 p.

Ouédraogo, H, A. (2006). L'impact de l'exploitation artisanale de l'or (orpaillage) sur la santé et l'environnement. Média terre. http://www.mediaterre.org/afriqueouest/actu, 20061121095625.html

Pecqueur B., et Zimmermann J.-B. (2004). « Les fondements d'une économie de proximités », in Pecqueur B., Zimmermann J.B. (dir.), Économie de proximités, HermèsLavoisier.

Père, M. (1992). «Vers la fin du mystère des ruines du Lobi ? Journal des Africanistes, 62 (1) : 79-93.

Rallet A., et Torre A. (2004). « Proximité et localisation ». Économie rurale n 280 : 2541 
Sachs, J. D. et Warner, A. M. (2001). "The curse of natural resources ». European economic review, 45(4) : 827-838.Sangaré O. (2016). Rôle de l'orpaillage dans le système d'activités des ménages en milieu agricole : cas de la commune rurale de Gbomblora dans la région sud-ouest du Burkina Faso. Québec, Université Laval, mémoire de maîtrise sur mesure en développement rural intégré.

Sawadogo, E. (2011). L'impact de l'exploitation artisanale de l'or : cas du site de Fofora dans la province du Poni (Mémoire de maîtrise, Université de Ouagadougou, Ouagadougou). Disponible sur le site :

http://www.memoireonline.com/01/12/5099/m L-impact-de-l-exploitationartisanale-de-l-or--cas-du-site-de-Fofora-dans-la-province-du-Poni.html

Schneider, K. (1993). " Extraction et traitement rituel de l'or ». Dans Fiéloux M., Lombard, J. et Kambou, F-J-M. (dir), Images d'Afrique et sciences sociales. Les pays lobi, birifor et dagara, Paris, Karthala/ORSTOM : 190-197.

Talbot D. (2008). «Les institutions créatrices de proximités ». Revue d'Économie régionale et Urbaine, $\mathrm{n}^{\circ} 3: 289$ - 310.

Talbot D., et Kirat Th. (dir.) (2005). « Proximité et institutions : nouveaux éclairages ». Économie et Institutions, 6-7 : 9-15.

Teschner, A. (2014). « Orpaillage pays for everything: How artisanal mining supported rural institutions following Mali's coup d'état ». Futures, Volume 62, Part A : 140-150.

Thune, M. (2011 «L'industrialisation de l'exploitation de l'or à Kalsaka, Burkina Faso : une chance pour une population rurale pauvre ? », EchoGéo [En ligne], 17 | 2011, URL : http://echogeo.revues.org/12535 ; DOI : 10.4000/echogeo.12535

Toé, D. (2013). État des lieux de l'orpaillage dans la région du Sud-ouest. Ouagadougou, École nationale des eaux et forêts, Rapport de stage de fin de cycle, diplôme de contrôleur des eaux et forêts, $61 \mathrm{p}$.

Tomicic, C., Vernez, D., Belem, T., et Berode, M. (2011). Human mercury exposure associated with small-scale gold mining in Burkina Faso. International archives of occupational and environmental health, 84 (5) : 539-546.

Torre A. (2004). «Introduction ». Économie rurale, Proximité et territoires, n $280: 2-7$.

Torre A. (2008). «Commentaire : réflexions à partir des textes de O. Bouba-Olga, M. Grossetti et D. Talbot. Proximité géographique et pragmatique de l'action ». Revue d'économie régionale et urbaine, $\mathrm{n}^{\circ} 3: 1-4$.

Torre A. (2010). «Jalons pour une analyse dynamique des proximités ». Revue d'économie régionale et urbaine, $\mathrm{n}^{\circ} 3: 409-437$.

Torre A., et Beuret J.-E. (2012). Proximités territoriales, Economica/Antropos.

Torre A., et Gilly J.P. (1999). « On the analytical dimension of Proximity Dynamics ». Regional Studies, 34 (2) : 169-180. 
Yaro, Y., Kaboré, I., et Kobanka, H. (2011). Étude sur le travail des enfants sur les sites d'orpaillage et les carrières artisanales dans cinq régions du Burkina Faso. Ministère de l'Action Sociale et de la Solidarité Nationale, rapport final. 85 p.

Werthmann, K. (2003). «Ils sont venus comme une nuée de sauterelles - Chercheurs d'or au Burkina Faso ». Dans R. Kuba, C. Lentz et C. N. Somda (dir.), Histoire du peuplement et relations interethniques au Burkina Faso, Paris, Karthala : 97-110.

World Bank. (2009). Mining together: Large-scale mining meets artisanal mining - a guide for action (Technical Report). World Bank.

Zimmerman J.B. (2008). «Le territoire dans l'analyse économique. Proximité géographique et proximité organisée ». Revue française de gestion, 4 (184) : 105 - 118.

\footnotetext{
i Oumar Sangaré est titulaire d'une maîtrise en développement rural intégré de l'université Laval, il est Chef de Service au Ministère de la Santé du Burkina Faso.

ii Patrick Mundler est professeur titulaire au département d'économie agroalimentaire et des sciences de la consommation de l'Université Laval.

iii Lala Safiatou Ouedraogo est doctorante en agroéconomie à l’Université Laval.
} 